\section{Management of Psuedomonas keratitis: A review article}

\section{Mouna Mohamad Al Saad ${ }^{1}$}

1 Faculty of Medicine, Department of special surgery/Division Ophthalmology. The University of Jordan, Amman, Jordan.

\section{Contact information:}

Dr. Mouna Mohamad Al Saad. factor in decreasing and preventing complication.

Pubmed and Medline were searched for articles related to Pseudomonas keratitis between year 2000 and 2017 to get current guidelines about the management of Pseudomonas keratitis. These articles are reviewed in this article and information related to management is summarized.

The most used agents to treat Pseudomonas are either aminoglycosides (usually gentamicin) fortified with a cephalosporin or mono therapy with a fluoroquinolones usually ciprofloxacin. In most areas, most strains of Pseudomonas were susceptible to ciprofloxacin. The role of topical steroids is discussed, as well as, available options for treatment of multidrug resistant Pseudomonas species.

Keywords

Psuedomonas, Keratitis, P. aeruginosa, Treatment.

\title{
Introduction
}

Bacterial keratitis is a vision threating infection characterized by epithelium loss, stromal infiltration by white blood cells and melting of the corneal stoma. It happens when one of the protective mechanisms of the ocular surface is sacrificed [1].

Early recognition and administration of appropriate choice of antibiotics is crucial if vision is to be restored. Pseudomonas species is commonly associated with contact lens wear [1-5]. Predisposing risk factors depend on the geographical locations and can be influenced by the penetration of contact lens. Trauma to the eye is an important risk factor in developing countries, meanwhile contact lens wear remains the main cause in developed countries [6]. A study from Malaysia suggested that Pseudomonas spp. is the main microorganism 
following vegetation related corneal injury is some regions. Pseudomonas group is a common inhabitant of soil, water and vegetation [7].

Other risk factors for bacterial keratitis include disease of the ocular surface and previous ocular surgeries [8].

Seasonal variation was noted in the presentation of Pseudomonas spp. keratitis, being mostly frequent in summer. Possibly warmer temperature, higher humidity and greater exposure to water may explain the higher incidence in summer [9].

\section{Biological characterization of Pseudomonas species}

Pseudomonas species is widespread in the environment and it can be isolated from various living sources, including plants, animals, and humans [10]. The organism is non-fermenting gram-negative, non-spore-forming, strictly aerobic, and catalase and oxidase positive. It can survive at temperature between 4 to $42^{\circ} \mathrm{C}$ but not at $\mathrm{pH}$ values below 4-5. Pseudomonas has also the ability to survive on minimal nutritional requirements and to tolerate a variety of physical conditions which allow it to persist in both community and hospital settings [10]. The organism forms biofilms on wet surfaces and in hospital environment such as sinks, humidifiers, and respiratory therapy equipment [11]. There are numerous Pseudomona s spp. which have been associated with opportunistic infections include $P$. aeruginosa, Pseudomonas maltophilia, P. fluorescens, P.putida, $P$. cepacia, $P$. stutzeri, and $P$. putrefaciens, but $P$. aeruginosa is the most common reported species causing human infection [12].

\section{Virulence of $\boldsymbol{P}$. aeruginosa}

Pseudomonas aeruginosa is a clinically important and opportunistic pathogen, often causing nosocomial infections. P. aeruginosa produces more than other species a variety of virulence factors, whose expression is regulated by different regulatory systems especially by Quorum sensing (QS) system. Most clinical isolated strains of $P$. aeruginosa can produce many virulence factors such as elastase, alginate, exotoxin $A$, exotoxin $S$, exotoxin $U$, alkaline protease and pyocyanin $[13,14]$. $P$. aeruginosa has a single polar flagellum that is responsible for motility. It is also involved in the adhesion to respiratory epithelial cells. This virulence effect induces an inflammatory response by interaction with receptor cells. $P$. aeruginosa synthesizes the exopolysaccharide alginate in response to environmental conditions. Alginate serves to protect the bacteria as biofilms in its surrounding environment and also enhances adhesion to solid surfaces. The organism can produce an alginate enzyme under certain infection conditions and both alginate biosynthetic and degradative enzymes are important for the development, maintenance and spread of $P$. aeruginosa biofilms in infected patients [14, 15].

\section{Antimicrobial resistance of $\boldsymbol{P}$. aeruginosa}

$P$. aeruginosa is the most common species and it colonizes more than $50 \%$ of hospitalized patients. $P$. aeruginosa is a leading cause of nosocomial infections, ranking second among the gram-negative pathogens reported to the National Nosocomial Infection Surveillance System in USA. The organism is responsible for severe nosocomial infections such as septicemia and pneumonia, life-threatening infections in immunocompromised persons, and chronic infections in cystic fibrosis patients [16].

A recent systemic review and meta-analysis study indicated that hospitalized patients with Multidrugresistant (MDR) $P$. aeruginosa infections appear to have increased mortality and hospital length [17, 18]. Infections caused by MDR $P$. aeruginosa are difficult to treat as the majority of isolates exhibit varying degrees of innate resistance or the constitutive expression of AmpC $\beta$ - lactamase and efflux pumps, combined with low permeability of the outer membrane. Acquired resistance is also reported due to the production of plasmid mediated AmpC beta ( $\beta$ )-lactamase, ESBLs and Metallo $\beta$-lactamase (MBL) enzymes [18]. Additionally, P. aeruginosa isolates from environment including water, sewage 
and human feces indicated the presence of high resistance rates to commonly used drugs in many Arab countries [19-23].

\section{Epidemiology $\boldsymbol{P}$. aeruginosa keratitis}

The occurring incidence rate of Pseudomonas aeruginosa keratitis was constant over the last 20 years at $1 / 2500$ of daily contact wearers and 1/500 of extended wear contact lens [6].

The incidence of Pseudomonas keratitis varies depending on geographical location. Template zones have a higher incidence rate of Gram positive bacteria causing keratitis and less aggressive disease [6]. However, Pseudomonas was found to be the most common Gran negative bacterial isolated in Toronto, Canada over the past 16 years [24] and in Western India with an incidence of $17.14 \%$ [1]. In Spain an incidence of $12.5 \%$ of all bacterial keratitis was reported and was found to be the most frequent Gram negative microorganisms [8]. In addition, this incidence was found to be $12.5 \%$ in China [2]. In Saudi Arabia, an incidence of $38.4 \%$ was reported [3].On the other hand, it has been reported that up to $80 \%$ of all positive cultures in Iran are caused by Pseudomonas spp. [4]. Moreover, P. aeruginosa was the most frequent bacteria isolated in contact Lens related ulcers with an incidence of $75 \%$ in France [5] and 79.7\% in Malaysia [7]. A higher percentage of contact lens related cases of Pseudomonas ulcers was found in a five year period from 2009 to 2014 in Texas U.S.A. [25].

Prognosis of bacterial keratitis depends on the distance from the limbus and the minimal inhibitory concentration (MIC) of the first antibiotic used or the lowest MIC if combined treatment is used [6].

The presence of conjunctival chemosis was found to be a clinical clue to the Pseudomonas as a causative agent in bacterial Keratitis [26]. Large ulcers are more likely to be caused by Pseudomonas [6].

The stain type of Pseudomonas aeruginosa (invasive or cytotoxic) may affect the clinical presentation, prognosis and response to antibiotics. It was suggested thatpseudomonas keratitis caused by in- vasive strains had better visual acuity than ones caused by cytotoxic strains. On the other hand, invasive ulcers are less significantly improved compared to cytotoxic ones at three months [27].

Risk factors for multiple and extremely drug resistance Pseudomonas include: bandage contact lens, topical steroids, previous corneal graft and ocular surface disease following Steven Johnson disease [28].

\section{Management of $\boldsymbol{P}$. aeruginosa keratitis}

As a rule, every case of suspected microbial keratitis should be scrapped for culture and sensitivity, especially with the increasing number of microbial resistant strains. Scrapping should be done with a surgical blade such as Bard Parker blade No.15, Kimora spatula or 21 gauge disposable needle. Samples should be sent immediately to bacteriology laboratory. The sample is normally inoculated on chocolate agar, blood agar, thioglycolate broth and incubated at $37^{\circ} \mathrm{C}$ for $24-48$ hours. Sabouraud agar plates should be also used for detection of yeast and rapid growing fungi. These plates should be kept at room temperature and $37^{\circ} \mathrm{C}$ for at least 4 days. Scrapping of small lesions (less than $2 \mathrm{~mm}^{2}$ ) is not advised and is not worthwhile. As a result, small lesions should be treated empirically. Scrapes should also be sent for preparing a Gram stains [6].

Clinical evaluation to antimicrobial response include: blurring of the margins to the infiltrates, decreased intensity of the infiltrates, decreased stromal edema and endothelial plaques, reduced anterior chamber reaction, re-epithelialization and cessation of corneal melting.

Topical antibiotics are used widely and empirically. Topical antibiotics remain the best treatment for bacterial keratitis. It was found that all frequently used antibiotics are equally effective, but the final outcome is not satisfactory because of corneal melting, scaring and perforation [29]. Treatment used varies depending on geographical location and available local antimicrobial susceptibility of Pseudomonas isolates. Monotherapy with ciprofloxacin 
of $0.3 \%$ (or other fluoroquinolones) is commonly used. Sub conjunctival injections of gentamicin may be also used. The combined use of two fortified antibiotics preparation of $1.5 \%$ gentamicin and $5 \%$ cefuroxime covers almost the entire spectrum of common causative bacterial pathogens [30]. Randomized clinical trials have demonstrated that monotherapy with fluoroquinolones is not inferior and has fewer side effects compared with combination treatment [31].

A study from Iran recommended the use of combination of ceftazidime and amikacin or ceftazidime and ciprofloxacin as the first treatment based on antibiotic sensitivity of isolated microbes. In that study it was shown that Pseudomonas isolates were resistant to chloramphenicol, trimethoprim, vancomycin and cefazolin. Moreover, these antibacterial agents should not be used as initial therapy in that region [32]. For example, In Australia, the major ocular pathogens are generally susceptible to the most commonly used antibiotics to treat microbial keratitis, and fluoroquinolones, aminoglycosides and cephalosporins is generally reserved for treatment of significant microbial keratitis [33].

In China, it was shown that most Gram-negative isolates including Pseudomonas was sensitive to neomycin but resistant to chloramphenicol [2]. A study from Iraq revealed that the use of ciprofloxacin alone as a starting treatment does not cover most of the bacteria causing superlative microbial keratitis. Another anti-microbial agent should be added. The choice of which treatment to be used depends on the likely microbial agent, taking into consideration the regional risk factors and clinical characteristics [30].

In Brazil, Pseudomonas isolates were susceptible to ciprofloxacin and ofloxcacin. One out of 239 cases was found to be resistant to gentamicin. Resistance to fourth generation fluoroquinolones was not observed [34]. In a recent study in South India, Pseudomonas isolates were found to be the second most common resistant bacterial isolate with resis- tance in bacterial Keratitis after Streptococcus pneumonia. The resistance was relatively uncommon and did not increase over 12 years (from 2002 to 2013) [35]. Data from Iran suggest that Pseudomonas isolates were mostly sensitive to ciprofloxacin followed by imipenem, meropenem and ceftazidime [4]. In Canada an increasing resistance to erythromycin $(p=0.018)$, ceftazidime $(P=0.046)$, piperacillin/tazobactam $(P=0.005)$ was seen in the past 16 years [21].

In Spain it is recommended to use aminoglycosides (gentamycin) and fluoroquinolones (ciprofloxacin) and avoid erythromycin due to increasing resistance to it [22].

A recent study evaluated the effect of different antibiotics on $P$. aeruginosa in vitro of primary human corneal fibroblasts. It was shown that the highest activity against planktonic pseudomonas was ciprofloxacin, Levofloxacin, and polymyxin B, followed by gentamicin and ofloxacin. Cefuroxime and chloramphenicol was found to be ineffective. It was also found that bactericidal and bacteriostatic antibiotics used in bacterial keratitis were unable to eradicate Pseudomonas infection of human fibroblasts culture in vitro. Their effectiveness depends on the cellular location of Pseudomonas [36].

According to the susceptibility of Pseudomonas strain the response to topical treatment may be affected. Adjuvant treatment with steroids, when used with moxifloxacin, was associated with better visual outcome in Pseudomonas keratitis caused by invasive strains compared to ones caused by cytotoxic strains. The size of scar at 3 months in the invasive strains was smaller when steroids and moxifloxacin were used compared to moxifloxacin alone. Less improvement was noted in the cytotoxic group $(p=0.07)[7,27]$.

Most ophthalmologists recommend starting anti-microbial empirically before culture results are available [37-39]. A study from Japan showed better results when anti-microbial agents were selected based on culture results, stressing the impor- 
tance of culture results [40]. However, a study from Oxford showed that $93.2 \%$ of bacteria isolated from infected corneas including Gram-positive and Gram-negative were sensitive to ciprofloxacin. On the other hand, $99.5 \%$ were sensitive to the combination of gentamicin and cefuroxime ( $P=0.0015)$. It was reported that there was no increase in the resistance over a decade, as indicated in the study period [41].

In some resistant cases, a combination of piperacillin/tazobactam may be useful [27]. The use of topical steroids (prednisone phosphate) combined with moxifloxacin did not improve the overall clinical outcome as showed in a multi-center clinical study that enrolled patients from India and USA [42].

In general, $P$. aeruginosa is mostly susceptible to fluoroquinolones, but multi drug resistant Pseudomonas strains are reported increasing in many country. For example, in Austria, strains are resistant to ciprofloxacin, gentamicin, tobramycin and amikacin but susceptible to ceftazidime, imipenem, meropenem and imipenim. It was also found that $P$. aeruginosa isolates has shown average susceptibility to piperacillin and ticarcillin [42].

Possible synergistic activity is documented between anti-microbial against Pseudomonas. Meropenem and ciprofloxacin with $90 \%$ of isolates showed synergistic or additive effect. This made combined anti- microbial a potential treatment against multi drug resistant strains [29]. Most common prescribed drugs are ciprofloxacin and tobramycin, a fortified aminoglycosides. Geographical location should be taken in consideration before giving them as treatment for $P$. aeruginosa [30].

Multi drug and extreme drug resistant Pseudomonas isolates are very difficult to treat. More than half of those patients required therapeutic grafts. Most sensitivity was reported to imipenem followed by colistin and neomycin. Few patients were sensitive to ceftazidime and azithromycin [25].

In advanced keratitis, continues infusion of topical antibiotics (ceftazidime $50 \mathrm{mg} / \mathrm{ml}$ ) with the
Morgan lens was effective when applied for one week followed by conventional topical antibiotics [44]. The early use of $19 \%$ topical colistin was found to be effective in the treatment of multi drug resistant Pseudomonas with few side effects [42]. Topical colitithemate of $1.6 \%$ maybe an effective topical alternative [43]. Corneal cross-linking is a promising new modality for non-healing corneal ulcers including ulcers secondary to Pseudomonas keratitis [47-48]. Amniotic membrane transplantation is effective for relieving pain, decreasing inflammation, stabilizing the cornea and promoting epithelial healing along with topical treatment [49]. It was found that double layered amniotic membrane transplantation had a good long term outcome in Pseudomonas infected corneas with descematocele. It reduced the risk of perforation and the need for emergent corneal grafting [50]. Salicylic acid may be beneficial as an anti-microbial agent in the treatment of Pseudomonas aeruginosa. It was shown that salicylic acid alters the membrane proteome of Pseudomonas aeruginosa, mildly increased the resistance to carbapenem antibiotics only with no effect on other antibiotics. Salicylic acid also reduces the clinical score and the number of bacteria with no effect on the number of neutrophils [51].

\section{Conclusion}

Pseudomonas, especially $P$. aeruginosa is mostly related to contact lens induced microbial keratitis.The most common used therapy is mono therapy with a fluoroquinolone or fortified aminoglycosides. Most strains are still susceptible to these antibiotics but geographical location should be taken in consideration. Multidrug resistant Pseudomonas isolates are observed in clinical practice and different treatment modalities are available. 


\section{References}

1. Kumar A, Pandya S, Kavathia G, Antala S, Madan M, JavdekarT. Microbial keratitis in Gujarat, Western India:finding 200 cases. Pan Arf med.J. 2011; 10:48-56.

2. Wang N, Huang Q, Tan Y, Lin L. Wu K. Bacterial Spectrum and Resistance Pattern in Corneal Infections at a Tertiary Eye Care center. Int. J. Ophthalmol 2016; 9(3):384-89.

3. Al Dahaheri HS, AlTamimi MD, Khandrkar RB, Khan M, Stone Du. Ocular Pathogens and Antibiotics Sensitivity in Bacterial Keratitis Isolates at King Khaled Specialist Hospital, 2011-2014. Cornea 2016; 35(6):789-794.

4. Hedayati H, Ghaderpanah M, Rasoulinejad M. Clinical Presentation and Antibiotic Susceptibility of Contact Lens Associated Microbial keratitis. J Ophtalmol 2015: article ID 152767.

5. Yildiz EH, Airiana S, Hammersmith KM, Rapuano CJ, Laibson PR, Verdi AS, et al. Trends in Contact Lens Related Corneal Ulcers at a Tertiary Referral Center. Cornea 2012; 31(10):1097-102.

6. Mark DP Willcox. Management and Treatment of Contact LensRelated Pseudomonas Keratitis. Clin Opthal 2012; 6 919-924.

7. Goh P P,ShamalaR,Chandamalar S, Tai X Y and National Eye Database Study Group. Contact Lens Related Corneal Ulcer: A Two Year Review. Med J Malaysia 2010; 65 suppl A: 120-123

8. Ruiz Caro JM, Cabrejas L,De Hoz MR, Mingo D, Duran SP. Clinical Features and Microbiological in Bacterial Keratitis in a Tertiary Referral Hospital. Arch Soc Esp Ophtalmol 2017; 92(9):419-425.

9. Gorski M, Genis A, Yushvayev S, Awwad A, Lazzaro DR. Seasonal Variation in the Presentation of Infectious Keratitis. Eye Contact Lens.2016; 42(5):295-7.

10. Lister PD, Wolter D J, Hanson N D. Antibacterial-Resistant Pseudomonas aeruginosa: Clinical Impact and Complex Regulation of Chromosomally Encoded Resistance Mechanisms. Clin Microbiol Rev 2009; 22(4): 582-610.

11. Leid JG, Willson CJ, Shirtliff ME, Hassett DJ, Parsek, MR, Jeffers AK.The exopolysaccharide alginate protects Pseudomonas aeruginosa biofilm bacteria from IFN-gamma-mediated macrophage killing. J Immunol 205; 175(11):7512-8.

12. Gellatly SL, Hancock RE. Pseudomonas aeruginosa: new insights into pathogenesis and host defenses. Pathol Dis 2013; 67, 159173.

13. Bradbury RS, Roddam LF, Merritt A, Reid D.W., Champion A.C. Virulence gene distribution in clinical, nosocomial and environmental isolates of Pseudomonas aeruginosa. J Med Microbiol 2010; 59:881-90.

14. Pereira SG1, Rosa, AC, Ferreira AS, Moreira LM, Proença, DN, Morais, PV, et al. Virulence factors and infection ability of Pseudomonas aeruginosa isolates from a hydropathic facility and respiratory infections. J Appl Microbiol 2014; 116(5):1359-68.

15. Mitov I., Strateva T, Markova B. Prevalence of virulence genes among Bulgarian nosocomial and cystic fibrosis isolates of Pseudomonas aeruginosa. Braz J Microbiol 2010; 41(3):588-95
16. Jones RN. Microbial Etiologies of Hospital-Acquired Bacterial Pneumonia and Ventilator-Associated Bacterial Pneumonia. Clin Infect Dis 2010; 51(1): S81-S87.

17. Hong DJ, Bae IK., Jang IH, Jeong SH., Kang HK, Lee K. Epidemiology and Characteristics of Metallo- $\beta$-LactamaseProducing Pseudomonas aeruginosa. Infect \& Chemother 2015; 47(2), 81-97.

18. Voor in 't holt AF, Severin JA, Lesaffre EM, Vos MC. A Systematic Review and Meta-Analyses Show that Carbapenem Use and Medical Devices Are the Leading Risk Factors for CarbapenemResistant Pseudomonas aeruginosa. Antimicrob. Agents Chemother 2014; 58 (5): 2626-2637

19. Shehabi AA, Haider AA., and Fayyad MK Frequency of antimicrobial resistance markers among Pseudomonas aeruginosa and Escherichia coli isolates from municipal sewage effluent water and patients in Jordan. IAJAA 2011; 1(1):1-8.

20. Shehabi AA, Masoud $H$, Balkam Maslamani FA. Common Antimicrobial Resistance Pattern, Biotypes and Serotypes Found among Pseudomonas aeruginosa Isolates from Patient's Stools and Drinking Water Sources in Jordan. J Chemother 2005; 17 (2): 179-183.

21. Al-Agamy MH, Shibl AM, Tawfik AF, Elkhizzi, NA, Livermore M. Extended spectrum metallo-beta lactamases among ceftazidime resistant Pseudomonas aeruginosa in Riyadh, Saudi Arabia. J Chemother 2012; 24(2): 97-100.

22. Al Bayssari C, Diene SM, Loucif L, Gupta SK, Dabboussi F, Mallat $\mathrm{H}$, et al. Emergence of VIM-2 and IMP-15 Carbapenemases and Inactivation of OprD Gene in Carbapenem-Resistant Pseudomonas aeruginosa Clinical Isolates from Lebanon. Antimicrob Agents Chemother 2014; 58(8): 4966-4970.

23. Mahfoud M, Al Najjar, M, Hamzeh, AR.(2015).Multidrug resistance in Pseudomonas aeruginosa isolated from nosocomial respiratory and urinary infections in Aleppo, Syria. J Infect Dev Ctries 2015; 19; 9(2):210-3.

24. Tam ALC, Cote $E$, Saldanha $M$, Lichtinger $A$ and Slomovic AR. Bacterial keratitis in Toronto: A 16-year review of the microorganisms isolated and the resistance patterns observed. Cornea 2017; 36(12):1528-1534.

25. Troung DT, Bui MT, Memon P and Cavanagh HD. Microbial keratitis at an Urban Public Hospital: A 10 year update. J Clin Exp Ophthalmol, 2015; 6(6):doi: 10.4172/2155-9570.100498.

26. Michael K.B, Rotchford A, Ramaesh K. Conjunctival Chemosis as a Specific Feature of Pseudomonas Aeruginosa Corneal Ulcers. Cornea, 2016 Sep.; 35(9):1182-1184.

27. Borkar DS, Fleiszig SM, Leong CBA, Lalitha P, Srinivasan M, Ghanekar A, et al. Association Between Cytotoxic and Invasive Pseudomonas Aeruginosa and Clinical Outcomes in Bacterial keratitis. JAMA Opthalmol 2013 131(2):147-153.

28. Fernandes M,Vira D., MedikonkaR and Kumar N. Extensively and Pan Drug Resistant Pseudomonas Aeruginosa Keratitis: Clinical Features, Risk Factors and Outcome. Graefes Arch Clin Exp Ophthalmol 2016; 254(2): 315-322. 
29. Austin A, Leitman T, and Rose-Nussbaumet J. Update On The Management of Infectious Keratitis. Ophthalmologists 2017; S0161-6420(16)32529-5.

30. Al Shakarachi Fl. Initial Therapy for Superlative Microbial Keratitis in Iraq.Br J. Ophthalmol 2007; 91:1583-1587.

31. Khokhar S, Sindhu N,Mirdha BP. Comparison of Topical 0,3\% Ciprofloxacin to Fortified Tobramycin-Cefazolin in the Theapy of Bacterial keratitis. Infection 2000; 28:149-152.

32. Mohammadpour M,Mohajernezhadfard Z, Khodabande A, Vahide P, Antibiotic Susceptibility Patterns of Pseudomonas Corneal Ulcers in Contact Lens Wearer.Middle East Afr. J Opthalmolol 2011; 18:228-231.

33. Willcox MD. Review of resistance of ocular isolates of Pseudomonas aeruginosa and staphylococci from keratitis to ciprofloxacin, gentamicin and cephalosporins. Clin Exp Optom 2011; 94(2):161-8.

34. Moriyama AS, Hofling Lima AL, Contact Lens Associated Microbial Keratitis. Arq Bras Ophtalmol, 2008; 71:32-36.

35. Lalitha P, ManoharanG,Karpagagam R, Pragna NV, Srinivasan M, Mascarenhas J, Das M, Porco TC, Lietman TM, Cavellos V and Keenan JD. Trends in Antibiotics Resistance in Bacterial Keratitis Isolated from South India. Br J Ophthalmolol. 2017 Feb; 10(12):108-113.

36. Del Mar Cendra M, Christodoulides M, Hossain P. Effect of Different Antibiotics Chemotheries on Pseudomonas Aeruginosa Infection in Vitro of Primary Human Corneal Fibroblast Cells. Front Microbiol 2017; 8:1614.

37. Palioura S, Henry C, Amescua G, Alfonso C. Role of steroids in the treatment of bacterial keratitis. Clinc Ohthalmolol 2016:10; 179-186.

38. Sy A, Srinivasan M, Mascarenhas J. et al. Puesdomonas Aeruginosa Keratitis Outcome and Response to Corticosteroid Treatment. Invest Opthalmol Vis sci. 2012; 53:267-272.

39. Borkar D, Acharya N, Leong C, Lalitha P, Srinivasan M, Oldenburg C, Cevallos V, Lietman T, Evans D, and Fleiszig. Cytotoxic Clinical Isolates of Psuedomonas Aeruginosa Identified During the Steroids for Corneal Ulcers Trial Show Elevated Resistance to Floroquinolones. BMC Ophthalmol 2014, 24; 14:54.

40. Uno T, Fukuda M, OhashiY, et al. Survey of Severe Contact Lens Associated Microbial Keratitis in Japan. Nihon Ganka Gakkai Zasshi 2011; 115:107-115.

41. Orlans HO, S. J. Hornby SJ, Bowler IC. In Vitro Antibiotic Susceptibility Patterns of Bacterial Keratitis Isolates in Oxford,UK: A 10-year review. Eye (Lond)2011; 25(4): 489-493.

42. Ku JY, Kim P. Tong J, Wechsler A. M.ccluskey P, Multi Resistant Pseudomonas Keratitis. Clin Exp Ophthalmol 2010: 38:818-819.

43. Hajoui FZ, DaoudiN, KabbajH, Alaoui A, Seffar M. Bacterialogical Study of the Stains Isolated From Ocular Infections. Ann Boil Clin (Paris) 2012; 70(6); 689-694.

44. Wang M, Smith WA, Duncan Jk and Miller JM. Treatment of Pseudomonas Keratitis by Continuous Infusion of Topical Antibiotics With the Morgan Lens. Cornea 2017; 36(5): 617620.
45. Jain R, Murthy SI, Motukupally SR, Jain MR. Use of Topical Colistin in The Treatment of Multiple Drug Resistant Pseudomonas Aeruginosa Bacterial Keratitis. Corea 2014; 33(9):923-927.

46. Chatterjee S. and Argawal D. Multi Drug Resistant Pseudomonas Aeruginosa Keratitis and its Effective Treatment with Topical Colistimethate. Indian J Ophthalmol. 2016; 64(2):153-157.

47. Zamani M, PanahiBazaz M, Assadi. Corneal Collagen Corneal Cross-linking for the Treatment of Non- healing Corneal Ulcers. J Opthalmic Vis Res 2015; 10 (1):16-20.

48. Khalil M, Jahad $H$, and Yasemi M. Collagen Cross-Linking for Treatment of Bacterial and Herpetic Keratitis. J Clin Diagn Res 2017; 11 (7) NC12-NC16.

49. Sheha H, Liang L, Tseng Li J, Schaffer CG. Suturesless Amniotic Membrane Transplantation for Severe Bacterial Keratitis. Cornea 2009; 28 (10):1118-1123.

50. Mohammadpour M. Sabet F. Long Term Outcomes of Amniotic Membrane Transplantation in Contact Lens Induced Pseudomonas Keratitis with Impending Corneal Perforation. J ophthalmic Vis Res 2016; 11(1): 37-41.

51. Bandara M, Sankaridurg P, Zhu H, Hume E, Willcox M. Effect of Salicylic Acid on the Membrane Prtoteome and Virulence of Pseudomonas Aeruginosa. Invest Ophalmol Vis Sci. 2016; 7(3):1213-220.

\section{Publish in The International}

\section{Arabic Journal of Antimicrobial Agents}

The Journal is an open access peer-reviewed journal that publishes scientific papers about all aspects of antimicrobials. The journal will publish original research articles, reviews, brief reports and case reports dealing with basic and clinical antibacterial agents, antiviral, antiprotozoals, antituberculuous, antifungal and antihelminthes agents. All manuscripts must be prepared in English, and are subject to a rigorous and fair peer-review process. Accepted papers will immediately appear online. The journal aims to advance the knowledge, attitude and the research of chemotherapy in the Arabic world in cooperation with international, national scientific and public societies as well as research centers with similar aims and objectives. 